\title{
CARACTERÍSTICAS DO FLUXO DE AR DE UM PULVERIZADOR HIDROPNEUMATICO PARA APLICAÇÃO DE AGROQUIMICOS EM PLANTAS ARBUSTIVAS
}

\author{
Gilton J. Rodrigues'; Maurí M. Teixeira²; Epídio I. F. Filho ${ }^{3}$ e Marcelo C. Picanço ${ }^{4}$
}

\section{RESUMO}

O transporte do ingrediente ativo até o interior do dossel da planta está condicionado à eficiência do equipamento utilizado. É importante estabelecer os principais parâmetros rastreáveis, que sirvam de base para avaliar e controlar as técnicas de pulverização visando à certificação da lavoura. Neste trabalho, foram estudadas as características do fluxo de ar de um pulverizador hidropneumático, usado em pulverização do cafeeiro. Foram avaliadas a uniformidade da velocidade e a vazão do ar na saída do difusor. A média da velocidade máxima do ar à saída do ventilador foi de $32,72 \mathrm{~m} \mathrm{~s}^{-1}$ e a média da mínima foi de $23,07 \mathrm{~m} \mathrm{~s}^{-1}$ às velocidades de 2.160 e $1.560 \mathrm{rpm}$ do rotor, respectivamente. 0 coeficiente de uniformidade da velocidade do ar na saída do ventilador situou-se entre 0,76 e 0,82. 0 lado direito apresentou, em média, uma vazão de ar $5 \%$ superior à do lado esquerdo.

Palavras-chave: tecnologia de aplicação, pulverização, agrotóxico, pulverizador hidropneumático.

\begin{abstract}
Air flow characteristics of a hydropneumatic sprayer for the agrochemical application in shrubs
\end{abstract}

The spray deposit of agrochemicals on the dorsal leaf surface of shrub canopy depends on the efficiency of the equipment used. It is important to establish the major traceable parameters that can be used to evaluate and control the spray techniques for crop certification. The air flow characteristics such as speed, flow rate and speed uniformity at the diffuser outlet, of a hydro-pneumatic sprayer used to spray coffee plants were evaluated. The mean maximum and minimum air speed at the fan outlet was $32.72 \mathrm{~ms}^{-1}$, and $23.07 \mathrm{~ms}^{-1}$, at the rotor speed of 2.160 and $1.560 \mathrm{rpm}$, respectively. The uniformity coefficient of the air speed at the fan outlet ranged from 0.76 to 0.82 . The mean air flow rate on the right side was $5 \%$ higher than on the left side.

Keywords: application technology, spray, agrochemicals, hydro-pneumatic sprayer.

\footnotetext{
Recebido para publicação em 19.07.2007

${ }^{1}$ Professor Adjunto, Universidade Federal Fluminense (UFF) pólo de Volta Redonda-R.J., giltongilton@ yahoo.com.br, fone:(24)33443026, fax (24) 33443019.

${ }^{2}$ Professor Adjunto, Universidade Federal de Viçosa,Viçosa-MG (UFV)

${ }^{3}$ Professor Adjunto, Universidade Federal de Viçosa,Viçosa-MG (UFV)

${ }^{4}$ Professor Adjunto, Universidade Federal de Viçosa,Viçosa-MG (UFV)
} 


\section{INTRODUÇÃO}

A eficácia de um tratamento depende da correta colocação do ingrediente ativo no alvo, no momento certo e na quantidade adequada. No entanto, na maioria das vezes, dá-se muito valor ao ingrediente ativo e pouco à tecnologia empregada, resultando uma aplicação insatisfatória e exigindo aplicações complementares ou a substituição do produto por outro mais eficaz.

A ineficácia das aplicações pode ser atribuída, em parte, à baixa penetração do líquido no dossel da planta, ao desvio da trajetória das gotas ou à ineficiência dos equipamentos. Na maioria das vezes, o transporte do ingrediente ativo para o interior do dossel da planta é condição básica para o controle eficaz de várias pragas e doenças.

$\mathrm{Na}$ pulverização, deve-se definir, criteriosamente, o tipo de alvo que se quer atingir. Cada cultura apresenta características próprias, devido à estrutura das plantas, idade, espaçamento e práticas culturais e ambientais. A eficiência da aplicação nos diferentes tipos de alvo requer equipamentos apropriados, doses adequadas com o mínimo de perda e de trabalho (HALL, 1993).

Independentemente do processo utilizado nas aplicações, é importante estabelecer os principais parâmetros, que sirvam de base para avaliar e controlar a técnica utilizada, garantindo, dessa forma, a qualidade do tratamento com o mínimo efeito danoso ao homem e ao ambiente.

O comportamento da corrente de ar produzida pelos pulverizadores hidropneumáticos tem sido alvo de pesquisa de inúmeros trabalhos, pois, ela é responsável pela condução e distribuição das gotas no alvo.

Segundo Chaim et al. (2004), alguns defeitos podem ser facilmente observados nos pulverizadores com ventiladores. A localização da tomada de ar situa-se, normalmente, na parte posterior do equipamento. Assim, o ar é sugado pelo ventilador depois da nuvem de gotas gerada pelos bicos. Conseqüentemente, grande parte das gotas poderia ser sugada pela hélice, com o deslocamento do trator, reduzindo-se a deposição na planta.

Kurdov e Kapitanov (1968) investigaram um critério prático para seleção de ventiladores para os pulverizadores pela configuração da saída do difusor. Esses autores estudaram a influência da largura de saída do ar, o efeito do defletor e a quantidade de aletas do ventilador no fluxo de ar e no seu alcance. Concluíram que, para garantir a velocidade de saída do ar de $36 \mathrm{~m} \mathrm{~s}^{-1}$, com fluxo na faixa de 13 a 15 $\mathrm{m}^{3} \mathrm{~s}^{-1}$, o maior alcance do ar foi obtido com a largura da saída do ventilador de 160 $\mathrm{mm}$, para a faixa compreendida entre 80 a $170 \mathrm{~mm}$.

Randall (1971), estudando o efeito do vento $e$ da velocidade de trabalho no volume de ar do ventilador, aplicou a teoria do fluxo plano turbulento de Abramovich (ABRAMOVICH, 1963). O autor demonstrou que, em um pulverizador com velocidade de saída do ar correspondente a $90 \mathrm{~m} \mathrm{~s}^{-1}$ e vazão de $1,53 \mathrm{~m}^{3} \mathrm{~s}^{-1}$, a velocidade decresce mais acentuadamente com o aumento da distância a partir da saída do difusor do que em um pulverizador usando a mesma quantidade de energia, porém com velocidade de saída equivalente a $41 \mathrm{~m} \mathrm{~s}^{-1}$ e vazão superior a $1,53 \mathrm{~m}^{3} \mathrm{~s}^{-1}$.

Os pulverizadores hidropneumáticos são amplamente utilizados nas pulverizações de plantas arbustivas. Esses equipamentos possuem dois sistemas principais: 0 hidráulico, para a formação das gotas que têm como unidade principal, o bico; e o ar, encarregado de transportar a gota até o alvo. Neste sistema é importante o estudo das características do ventilador, por ser responsável pela produção de ar e também ser o maior consumidor de energia.

O objetivo deste trabalho foi avaliar um pulverizador hidropneumático, utilizado para aplicação de inseticidas na cultura do café, com diferentes velocidades de trabalho do ventilador, a fim de determinar os principais parâmetros da corrente de ar. 


\section{MATERIAL E MÉTODOS}

Os ensaios foram realizados no Laboratório de Mecanização Agrícola do Departamento de Engenharia Agrícola da Universidade Federal de Viçosa-UFV. O teste consistiu em avaliar as características da corrente de ar produzida pelo ventilador de um pulverizador hidropneumático, marca Hatsuta, modelo SS-420, acoplado a um trator Valtra Valmet, modelo 800 L (Figura 1), ambos de propriedade da UFV. Esse tipo de equipamento hidropneumático apresenta baixa vazão de ar, sendo amplamente utilizado para aplicação de agroquímicos na cultura do café.

As medições foram feitas com o pulverizador nivelado, em local fechado com paredes laterais a $15 \mathrm{~m}$ de distância do local de trabalho. A temperatura e umidade relativa do ambiente foram medidas usando-se um termohigrômetro, enquanto a velocidade do ar foi medida com um anemômetro de paletas, com escala de medição de 0,3 a $40 \mathrm{~m} \mathrm{~s}^{-1}$ e erro de $\pm 3 \%$.
A velocidade angular do ventilador foi medida, no cento do rotor, utilizando-se um tacômetro digital. As variáveis avaliadas, medidas na saída do difusor, foram a velocidade do ar ( $\square$, vazão do ar $(Q)$ e uniformidade da velocidade do $\operatorname{ar}\left(\eta_{1}\right)$. Para tal, foram utilizadas cinco velocidades angulares do rotor: 2.160 rpm, rotação máxima recomendada pelo fabricante, e 1.960, 1.860, 1.760 e 1.560 rpm.

Os ensaios de laboratório foram realizados, segundo a metodologia proposta por Sotolongo e Herrera (1986).

As medições da velocidade do ar foram realizadas em toda a periferia da saída do ventilador, em 80 pontos, segundo o esquema apresentado na Figura 2. Cada ponto corresponde a uma área retangular determinada, ou seja, marcaram-se 16 pontos distribuídos na periferia do ventilador, sendo que em cada um, foram estabelecidos cinco pontos eqüidistantes (nomeados a, b, c, d, e), cobrindo toda a largura da saída do difusor. Em cada ponto, as medições foram repetidas três vezes.

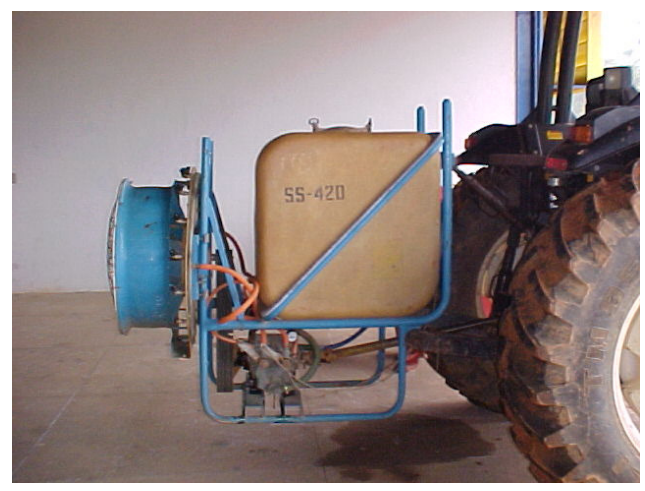

Figura 1. Conjunto trator/pulverizador utilizado nos ensaios do fluxo de ar.

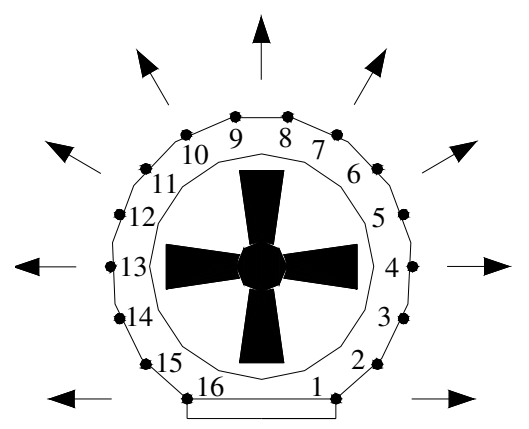

(a)

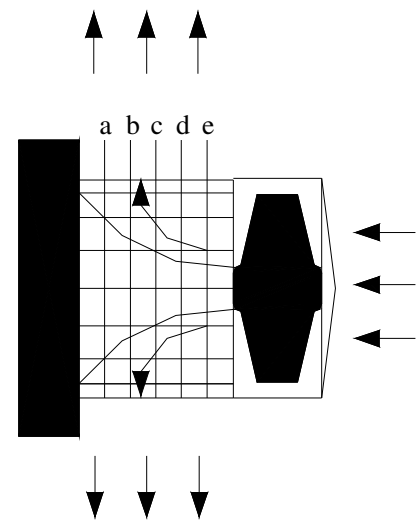

(b)

Figura 2. Pontos de medição da velocidade do ar na saída do difusor do ventilador - vistas frontal $(A)$ e lateral $(B)$. 
Tabela 1. Características técnicas do anemômetro

\begin{tabular}{ll}
\hline Parâmetros fornecidos & Escala de leitura \\
\hline Constante de tempo & $10 \mathrm{~s}$. \\
Velocidade & 0,125 a $50,0 \mathrm{~m} \mathrm{~s}^{-1}$ \\
Leitura de velocidade & 0 a $50 \mathrm{~m} \mathrm{~s}^{-1}$ \\
Exatidão & $\pm 0,5 \%$ da escala selecionada \\
Repetitividade & $< \pm 1,0 \%$ da leitura \\
Tempo de resposta ao fluxo & $0,2 \mathrm{~s}$. \\
Operação do sensor & 0 a $93^{\circ} \mathrm{C}$ \\
\hline
\end{tabular}

A medição da velocidade do ar foi efetuada com um anemômetro, cujo princípio é a transdutância. Suas características são apresentadas na Tabela 1.

A vazão de ar (Q) foi determinada por meio da Equação 1.

\section{Erro! Indicador não definido.} (1)

em que:

$\mathrm{Q}=$ vazão de $\operatorname{ar}, \mathrm{m}^{3} \mathrm{~s}^{-1}$;

$a_{i}=$ áreas da seção onde se realizam as medições, $\mathrm{m}^{2}$; e

$\omega_{0 \mathrm{i}}=$ velocidade em cada ponto, $\mathrm{m} \mathrm{s}^{-1}$.

O coeficiente de uniformidade do ar representa a distribuição da velocidade na saída do ventilador (Figura 3 ). A variação se deve a fatores como o desenho do difusor, a existência ou não de defletores e a proximidade da parede do ventilador. Para isso, estabelece-se um fator que quantifica este parâmetro, conforme Equação 2 (BRAZEE, et al., 1981).

$\eta_{1}=\int_{0}^{B_{o m}} \frac{\omega_{0}}{\omega_{0 m}} \frac{\partial B o m}{B_{0 m}}$

em que:

$\eta_{1}=$ coeficiente de uniformidade da velocidade do ar;

$\omega_{0}=$ velocidade do ar em cada ponto da abertura da saída do ventilador, $\mathrm{m} \mathrm{s}^{-1}$;

$\omega_{0 \mathrm{~m}}=$ velocidade máxima do ar na seção, $\mathrm{m} \mathrm{s}^{-1}$;

$\mathrm{B}_{0 \mathrm{~m}}=$ largura de saída, m; e

$\partial=$ diferencial de integração.

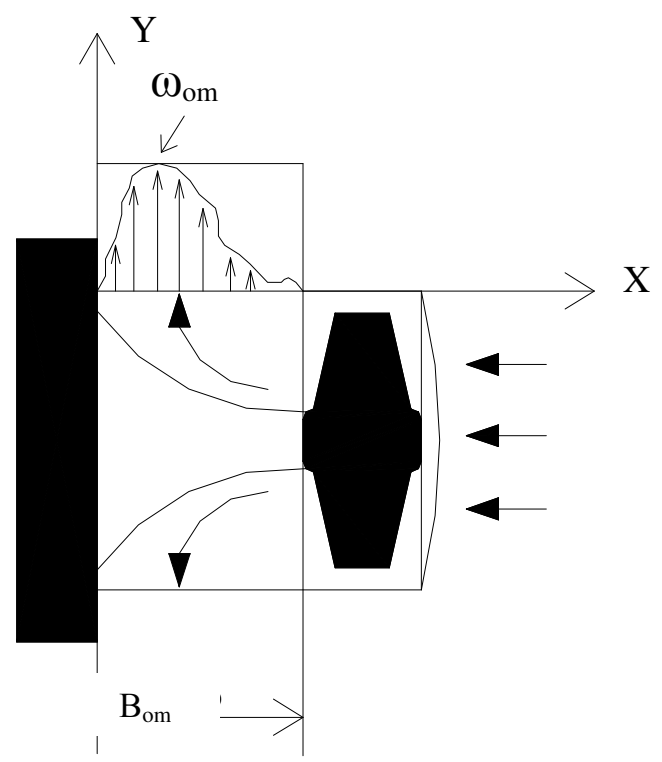

Figura 3. Esquema da velocidade do ar na periferia do ventilador, após a passagem pelo rotor (vista de cima). 


\section{RESULTADOS E DISCUSSÃO}

A Tabela 2 apresenta a velocidade média do ar nas rotações utilizadas.

A média da velocidade máxima foi de $32,72 \mathrm{~m} \mathrm{~s}^{-1}$ na rotação de $2.160 \mathrm{rpm}$, decaindo, gradativamente, com a diminuição na velocidade do rotor, chegando à média mínima de $23,07 \mathrm{~m} \mathrm{~s}^{-1}$ para a rotação de $1.560 \mathrm{rpm}$.

A distribuição radial da velocidade do ar (Figura 2) é representada nas figuras 4 a 8.

$\mathrm{Na}$ seção "a", a mais afastada do ventilador, os valores de velocidade foram maiores que nas outras; por outro lado, na seção "e", a mais próxima do rotor, as velocidades do ar foram menores, com diferenças variando de 5 a $8 \mathrm{~m} \mathrm{~s}^{-1}$ com o rotor a $2.160 \mathrm{rpm}$ (Figura 4). Tal fato ocorre devido à mudança brusca na direção do ar dentro do difusor, passando de axial para radial, provocando inércia do ar ao manter o fluxo, mudando de sentido. Este fenômeno influencia o coeficiente de uniformidade da velocidade de saída e, posteriormente, a formação da corrente de ar.

Nos pontos 1 e 16, as velocidades do ar foram maiores, o que se deve ao fato de o ventilador não ter saída pela parte inferior, aumentando a velocidade nesses pontos.

A velocidade do ar foi maior no lado direito do ventilador, nos pontos de medição de número 1 a 8 , em média de $6 \mathrm{~m} \mathrm{~s}^{-1}$. Este fenômeno está relacionado ao sentido horário de rotação do ventilador e ao impedimento do escoamento do ar pela parte inferior do difusor, provocando uma diferença no escoamento de ar em ambos os lados do ventilador, conforme reportado por Herrera e De las Cuevas (2002).

$\mathrm{Na}$ parte superior do ventilador, entre os pontos 7 e 11, houve tendência à diminuição na velocidade do ar, devido ao desenho do equipamento.

Nas demais rotações do rotor, o comportamento da velocidade do ar foi semelhante, diminuindo, apenas, a velocidade média com a diminuição na rotação (Figuras 5 a 8).

Tabela 2. Velocidade média do ar do ventilador em distintos regimes de rotação do rotor

\begin{tabular}{cc}
\hline Freqüência $(\mathrm{rpm})$ & Velocidade do $\operatorname{ar}\left(\mathrm{m} \mathrm{s}^{-1}\right)$ \\
\hline 1500 & 23,07 \\
1760 & 26,22 \\
1860 & 28,75 \\
1960 & 29,55 \\
2160 & 32,73 \\
\hline
\end{tabular}

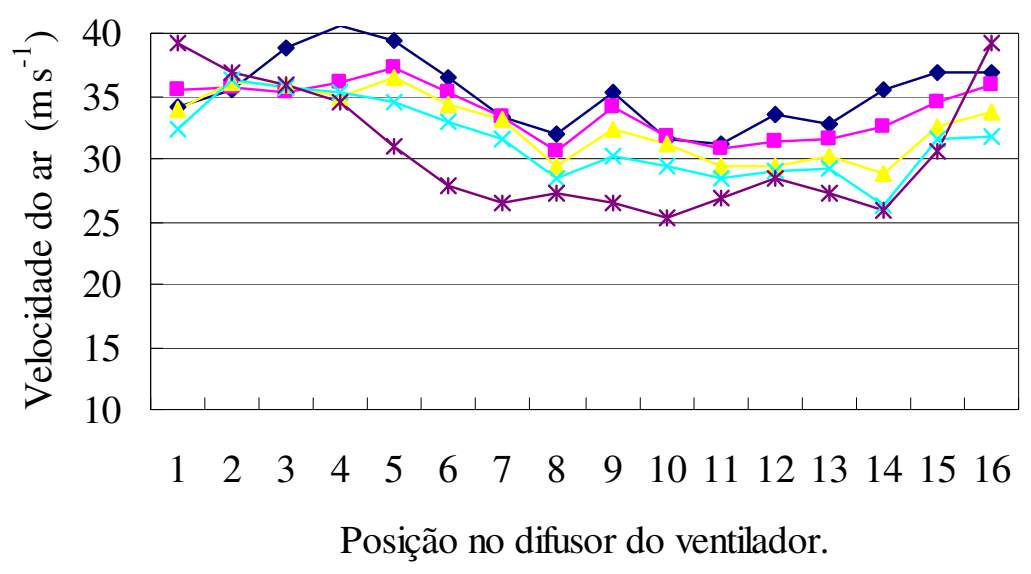

$\rightarrow \mathrm{a} \rightarrow-\mathrm{b} \leadsto \mathrm{c} \rightarrow \mathrm{d} \rightarrow \mathrm{e}$

Figura 4. Velocidade do ar na saída do ventilador, nas seções a, b, c, d, e, com a velocidade do rotor de $2.160 \mathrm{rpm}$. 


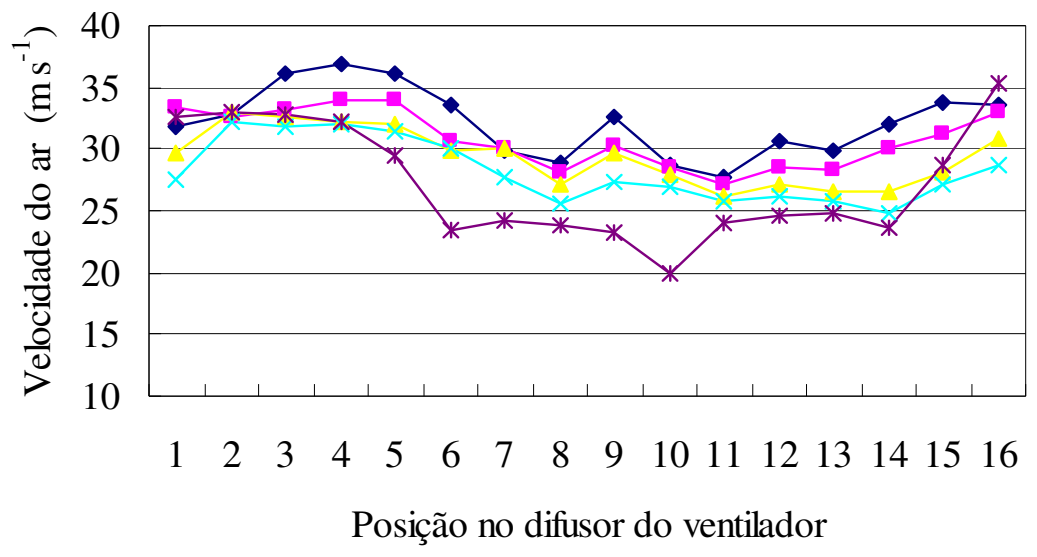

$\multimap \mathrm{a} \longrightarrow \mathrm{b} \longrightarrow \mathrm{c} \rightarrow \mathrm{d} \rightarrow \mathrm{e}$

Figura 5. Velocidade do ar na saída do ventilador, nas seções a, b, c, d, e, com a velocidade do rotor de $1.960 \mathrm{rpm}$.

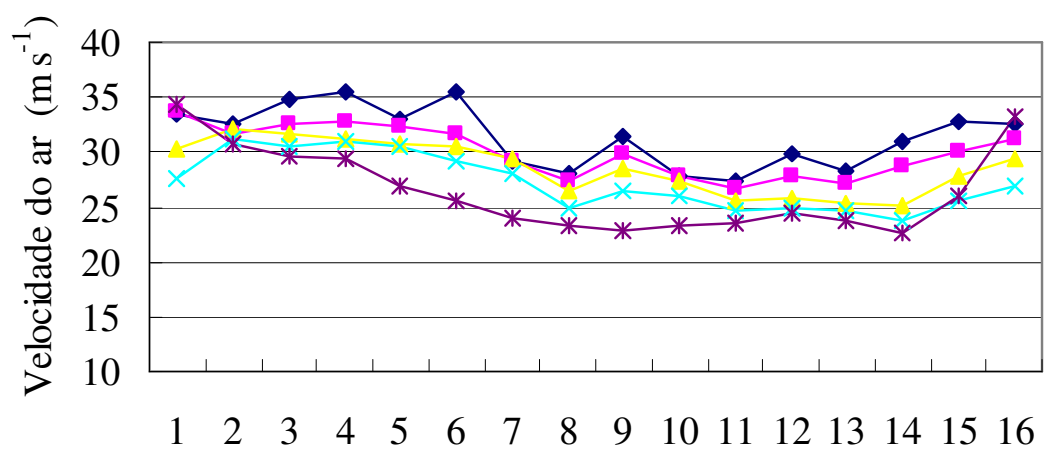

Posição no difusor do ventilador

$$
\multimap \mathrm{a} \rightarrow-\mathrm{b} \rightarrow \mathrm{c} \rightarrow \mathrm{d} \rightarrow \mathrm{e}
$$

Figura 6. Velocidade do ar na saída do ventilador, nas seções a, b, c, d, e, com a velocidade do rotor de $1.860 \mathrm{rpm}$.

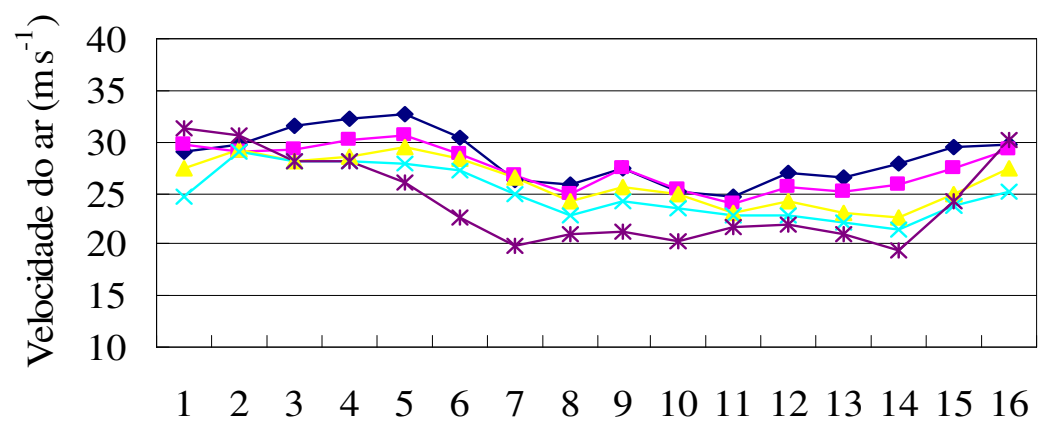

Posição no difusor do ventilador

$$
\multimap \mathrm{a} \rightarrow-\mathrm{b} \rightarrow \mathrm{c} \rightarrow \mathrm{d} \rightarrow \mathrm{e}
$$

Figura 7. Velocidade do ar na saída do ventilador nas seções a, b, c, d, e, com a velocidade do rotor de $1.760 \mathrm{rpm}$. 


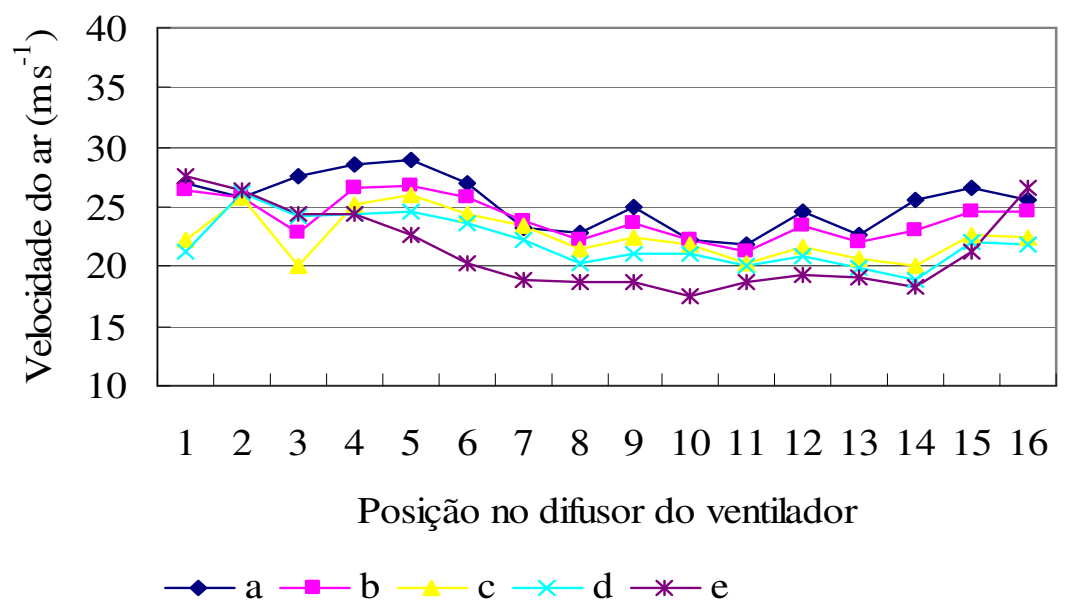

Figura 8. Velocidade do ar na saída do ventilador nas seções a, b, c, d, e, com a velocidade do rotor de $1.560 \mathrm{rpm}$.

Tabela 3. Vazão de ar proporcionado pelo ventilador em diferentes rotações

\begin{tabular}{cccc}
\hline Rotação $(\mathrm{rpm})$ & \multicolumn{3}{c}{ Vazão do ventilador $\left(\mathrm{m}^{3} \mathrm{~s}^{-1}\right)$} \\
\hline & Lado direito & Lado esquerdo & Total \\
\hline 1560 & 1,85 & 1,66 & 3,52 \\
1760 & 2,12 & 1,88 & 4,00 \\
1860 & 2,32 & 2,06 & 4,39 \\
1960 & 2,37 & 2,14 & 4,51 \\
2160 & 2,62 & 2,37 & 5,00 \\
\hline
\end{tabular}

Na rotação de 2.160 rpm, a vazão total foi de $5,0 \mathrm{~m}^{3} \mathrm{~s}^{-1}$ (Tabela 3). O lado direito apresentou, em média, uma vazão $5 \%$ superior à do lado esquerdo, nas cinco rotações. Esta diferença é baixa, não afetando a qualidade da cobertura da árvore pela corrente de ar. Contudo, considerando-se que a variação na velocidade angular do ventilador influencia a corrente de ar, esta tem efeito na distribuição do agrotóxico na planta, que é um importante fator a ser analisado (SOTOLONGO e HERRERA, 1986).

O coeficiente de uniformidade representa a distribuição da velocidade do ar ao sair pelo difusor do ventilador. O gráfico da Figura 9 representa o valor deste coeficiente para os 16 pontos, medidos nas cinco rotações. Em geral, o comportamento foi similar nas cinco rotações. No caso de 1.760 rpm, a curva não coincide com os demais, em alguns pontos. Os valores se situam entre o máximo de 0,82 e o mínimo de 0,76. Esta faixa de valores é considera boa para esse tipo de equipamento, uma vez que o máximo alcançado em equipamentos comerciais é 0,835 (BRAZEE, et al., 1981).

Comparando o lado direito (1 ao 8) do pulverizador com o esquerdo (9 ao 16), observase uma tendência a maiores valores do coeficiente de uniformidade no primeiro. Tal fato coincide com o lado, onde ocorre maior vazão de ar, donde infere-se que existe uma relação com o aumento do coeficiente, ao ocupar a saída do difusor, com maior vazão de ar.

O maior coeficiente $(0,82)$ ocorreu no ponto 2 . Este representa uma posição próxima à zona inferior do difusor, que ficou sob influência do ar, que não sai pela parte inferior do ventilador, sendo forçado a sair por esse lugar pela rotação do ventilador. O menor coeficiente $(0,71)$ ocorreu no ponto 14 , no lado esquerdo do ventilador, que coincide com a zona de menor fluxo de ar.

Os pontos de menor fluxo de ar apresentam, também, o menor coeficiente de uniformidade. Uma possível solução seria dotar o ventilador de defletores com o redirecionamento do ar a fim de uniformizar o fluxo em toda a extensão de saída do rotor. 


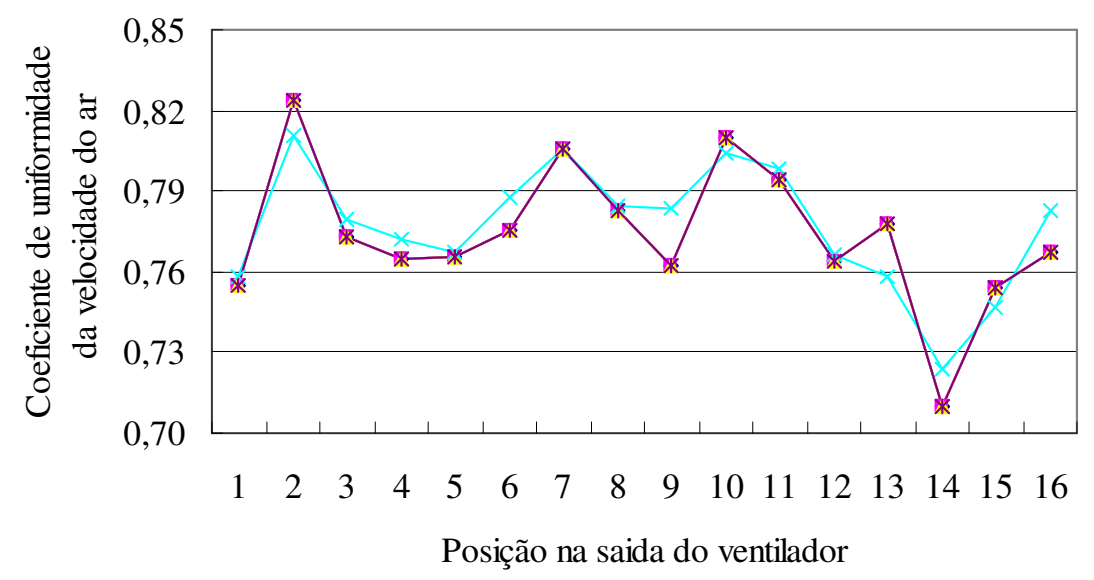

$\rightarrow-2160 \mathrm{rpm} \rightarrow-1960 \mathrm{rpm} \leadsto 1860 \mathrm{rpm} \leftarrow 1760 \mathrm{rpm} \rightarrow 1560 \mathrm{rpm}$

Figura 9. Coeficiente de uniformidade da velocidade do ar na saída do ventilador em cinco rotações do rotor.

Tabela 4. Coeficiente de uniformidade média na saída do ventilador

\begin{tabular}{ccccccc}
\hline Rotação (rpm) & \multicolumn{2}{c}{ Lado direito } & \multicolumn{2}{c}{ Lado Esquerdo } & Geral \\
\cline { 1 - 5 } & Uniformidade & Desv.pad & Uniformidade & Desv.pad & \\
\cline { 1 - 5 } 2160 & 0,79 & 0,02 & 0,77 & 0,03 & 0,78 \\
1960 & 0,79 & 0,02 & 0,77 & 0,03 & 0,78 \\
1860 & 0,79 & 0,02 & 0,77 & 0,03 & 0,78 \\
1760 & 0,78 & 0,02 & 0,77 & 0,03 & 0,78 \\
1560 & 0,79 & 0,02 & 0,77 & 0,03 & 0,78 \\
\hline
\end{tabular}

$\mathrm{Na}$ Tabela 4, observam-se as médias do coeficiente de uniformidade da saída do ar para o lado direito e esquerdo e total do ventilador. Constata-se um coeficiente médio de 0,78 para todas as rotações do rotor. Tal fato demonstra que este coeficiente depende, fundamentalmente, do desenho do difusor e da relação da largura de saída com a quantidade de ar que o rotor impulsiona. Conforme comentado, trata-se de um valor ótimo para este pulverizador comercial.

No lado direito do ventilador, o coeficiente foi de 0,79 em todas as rotações, exceto a $1.760 \mathrm{rpm}$, que foi de 0,78 , enquanto no lado esquerdo, o valor foi de $0,77 \mathrm{em}$ todas as rotações do rotor, ficando o valor médio em 0,78 . Em geral, o valor do coeficiente de uniformidade do lado direito é maior que o esquerdo em 0,02.

\section{CONCLUSÕES}

- O coeficiente médio de uniformidade da velocidade do ar na saída do ventilador é compatível com as exigências para os ventiladores comerciais.

- O lado direito apresentou, em média, uma vazão de ar $5 \%$ superior ao lado esquerdo.

- Houve diferença da velocidade do ar, na seção de saída do ventilador, de acordo com a posição em relação ao rotor, atingindo valores maiores nas seções mais distantes.

\section{BIBLIOGRAFIA}

ABRAMOVICH, G.N. The Theory of turbulent jets. Fitzmatgiz.(ed) Schindel, L.H. MIT.Press. 1963, 671p. 
BRAZEE, R.D.; FOX, R.D.; REICHARD, D.L.; Hall, F.R.. Turbulent Jet Theory Applied to air sprayers. Transactions of the ASAE, St. Joseph. v.24 n. 2. p. 266-272, 1981.

CHAIM, A.; PESSOA, M.C.P.Y.; FERRACINI, V.L. Eficiência de deposição de pulverização em videiras, comparando bicos e pulverizadores. Pesticidas: R. Ecotoxol. e Meio Ambiente, Curitiba, v. 14. p. 39-46, 2004

HALL, F. Application to plantation crops.In: Application technology for crop protection. Cab International. Walligford 1993. p. 187-211.

HERRERA, M.I ; De las CUEVAS, H.M. Analisis de la velocidad del aire en la corriente de las asperjadoras utilizadas en frutales. Revista Ciencias Técnicas
Agropecuarias. La Habana, Cuba v. 2 n.2. p.151-162, 2002.

KURDOV, Z.; KAPITANOV, D. Resultado de algunas investigaciones sobre los ventiladores axiales y radiales para asperjadoras de tractor. Sieljoztopanska Tejnika.Moscú. v. 5. p. 105-114. 1968.

RANDALL, J.M. The relationship between air volumen and pressure on spray distribution on fruit trees. Journal Agric. Engr. Res. v. 16 n.1. p. 1-31, 1971.

SOTOLONGO, I.; HERRERA, M. Caracterización de la corriente de aire de una asperjadora de ventilador en el plano horizontal.

Ciencias

Técnicas

Agropecuarias. La Habana, Cuba v. 1, p. 112. 1986. 